\title{
Revisión
}

\section{Las fiebres manchadas y su importancia en Costa Rica}

\section{(Spotted fever and their importance in Costa Rica)}

\author{
Laya Hun-Opfer
}

\section{Resumen}

La Fiebre Manchada de la Montañas Rocosas es una zoonosis transmitida por garrapatas y causada por una bacteria intracelular obligatoria, la Rickettsia rickettsii. Es una enfermedad sistémica, de moderada a severa, producida por la infección del endotelio vascular de pequeños vasos de la mayoría de órganos y tejidos. Es la más letal de las infecciones transmitidas por garrapatas y desde su descubrimiento, hace cerca de 100 años, todavía se presenta, aunque esporádicamente, de forma persistente, y a pesar de que se cuenta con antibióticos efectivos, la mortalidad sigue siendo de mas del $10 \%$ y los pacientes requieren terapia intensiva durante la infección si esta no es diagnosticada y tratada a tiempo. En tiempo recientemente se han descrito otras especies de garrapatas que pueden transmitir rickettsias a humanos así como nuevas especies de rickettsias que pueden producir cuadros de fiebres manchadas y aunque se ha dilucidado parte de sus mecanismos patogénicos, persisten muchas dudas respecto a su virulencia. Desde 1975 se han reportado brotes de esta enfermedad en Costa Rica y el agente etiológico causal, la Rickettsia rickettsii, fue aislada e identificada en la mayoría de los casos en el Laboratorio de Virología de la Facultad de Microbiología de la Universidad de Costa Rica.

Descriptores: Rickettsia rickettsii, Fiebre Manchada de las Montañas Rocosas, patogénesis, virulencia, diagnóstico de laboratorio, epidemiología, vectores, Costa Rica

Centro de Investigaciones en EnfermedadesTropicales (CIET). Facultad de Microbiología. Universidad de Costa Rica

Abreviaturas: CDC, Centro de enfermedades infecciosas; FMMR, Fiebre Manchada de las Montañas Rocosas; IFA, Inmunofluorescencia; IFN$\gamma$, Interferón gamma; IL-I $\beta$, Interleucina -I betha; PCR, Reacción en Cadena de la Polimerasa; TNFá, Factor de necrosis tumoral alpha. Correspondencia: Laya Hun. rlhun@cariari.ucr.ac.cr

ISSN 0001-6002/2008/50/2/77-86 Acta Médica Costarricense, (O2008 Colegio de Médicos y Cirujanos

\section{Abstract}

Rocky Mountain Spotted fever is a tick vector disease caused by an obligated intracellular bacteria, Rickettsia rickettsii. It is a severe systemic illness caused by the infection of endothelial cells of small vessels of most organs and tissues of the human body. It is the most lethal tick-born disease and since its discovery more than 100 years ago it still appears sporadically, but persistently. Although there is treatment with effective antibiotics, mortality rates are near $10 \%$ and infected patients require intensive care when the illness is misdiagnosed and there is a delay in treatment. Other species of ticks and new species of rickettsia have been described recently related to Spotted Fever diseases and although the pathogenesis of the disease has been dilucidated in part, there are a lot of questions concerning the mechanisms of virulence.

Key words: Richettsia rickettsii, Rocky mountain spotted fever, pathogenesis, virulence, diagnosis, epidemiology, insect vectors, Costa Rica.

Recibido: 21 de agosto de 2007 Aceptado: 9 de octubre de 2007 
La Fiebre Manchada de las Montañas Rocosas (FMMR) es una enfermedad febril cuyas importancia y severidad fueron establecidas hace cerca de un siglo, y es considerada hasta la fecha la causa de muerte más común entre las infecciones transmitidas por artrópodos en los Estados Unidos. La alta mortalidad $^{1}(25 \%)$ ocurre principalmente por una falla en el diagnóstico clínico y, en consecuencia, por falta de tratamiento con el antibiótico adecuado. Esta rickettsiosis es característica del hemisferio occidental y se ha descrito en países como: México, Canadá, Panamá Colombia, Brasil y Argentina. ${ }^{2}$ En Costa Rica se describió la presencia del agente etiológico por primera vez en $1975^{3}$ y desde entonces se han reportado pequeños brotes en el país, con tasas de mortalidad muy altas en los pacientes infectados.

Las rickettsias son microorganismos que se pueden observar al microscopio de luz como cocobacilos pleomórficos; se multiplican únicamente dentro de algunas células de animales susceptibles, por lo tanto, son parásitos obligados.

Las rickettsias pertenecen a una subdivisión de las proteobacterias del orden Rickettsiales, familia Rickettsiaceae, tribu Rickettsieae, género Rickettsia. Una característica que las distingue de las demás bacterias es que son transmitidas por artrópodos en condiciones naturales, y por esta razón las dividieron en 2 grupos con base en las manifestaciones clínicas, los aspectos epidemiológicos y las características inmunológicas. 1) Grupo Tifo: a) Tifus Epidémico- Rickettsia prowazeki transmitida por piojos, b) Tifus endémico-Rickettsia typhi transmitida por pulgas, 2) Grupo de las Fiebres Manchadas: la más importante en el continente americano es la Fiebre Manchada de las Montañas Rocosas (FMMR)-Rickettsia rickettsii transmitida por garrapatas. $^{4}$

\section{Aspectos históricos de la FMMR}

En 1883 un médico en Idaho, EEUU, describió una forma de sarampión "negro" en la rivera del río Snake Valley. La enfermedad como "fiebres manchadas", no aparece en la bibliografía médica sino hasta 1899, cuando Maxey describe una enfermedad febril con delirio y exantema (rash) que se presenta en la piel como rojo-morado-negro, y que aparece primero en tobillos, muñecas y la frente, con una diseminación general muy rápida. Su incidencia era esporádica, más común en la primavera, y la opinión general era que se transmitía por el agua potable del deshielo de la nieve. ${ }^{5}$

Earl Strain, un médico familiar de Montana sugiere que el vector es la garrapata del ganado, tomando como analogía los estudios de Walter Reed y de Theobold Smith, de la transmisión de la fiebre amarilla y de la fiebre del ganado en Texas, respectivamente. Wilson y Chowning sugieren a la garrapata de la madera como el vector por los estudios de campo y proponen que el parásito eritrocitario Piroplasma hominis es el agente causal.
Howard Taylor Ricketts (1911),,6 un científico de Chicago, entra al Bitter Rock Valley, en Montana, sin prejuicios en favor o en contra de los conceptos prevalentes. En una serie de estudios realizados entre 1906 y 1910, Ricketts transmitió la enfermedad a conejillos de indias $(C$. porcellus) y a monos (Macacus rhesus) inoculando la sangre de pacientes con FMMR, y demostró la transmisión de la enfermedad de animal a animal por la garrapata de la madera, Dermacentor andersoni, con lo que evidenció, además, la infección natural en estas garrapatas. La sangre de los animales que sobrevivían a la infección resultó protectora de otros animales infectados, si se inoculaba varios días antes de la infección. ${ }^{5-7}$

Ricketts demostró pequeños cuerpos tipo bacterias en los tejidos de las garrapatas y que las hembras infectadas, transmitían el agente transováricamente a algunos de los huevecillos. Las garrapatas infectadas, sea en huevo o en cualquiera de los estadios del ciclo de desarrollo, cuando se alimentan de mamíferos infectados mantienen al microorganismo de por vida. Junto a su colega Wilder, comprobaron mediante estudios serológicos cruzados, que la FMMR y la fiebre del tifo son dos entidades diferentes. ${ }^{6,7}$ Trágicamente, el Dr. Ricketts murió de tifus en México en 1910, mientras estudiaba la enfermedad.

Da Rocha - Lima ${ }^{8}$ honra a Ricketts y propone el nombre de Rickettsia para estos agentes que causan el tifo y las fiebres manchadas.

En un estudio independiente, del cual Ricketts no tenía conocimiento, McCalla y Brereton, ${ }^{9}$ de Idaho, transmitieron la fiebre manchada a dos humanos utilizando una garrapata de uno de sus pacientes.

Wolbach demostró la multiplicación intracelular de las rickettsias en los tejidos de las garrapatas.

Spencer y Parker, en sus estudios de ecología y control, infectan conejillos de indias por inyección intraperitoneal de tejidos de garrapatas. Al advertir que el suero de los pacientes protegía a los cuilos infectados, Cox desarrolló la primera vacuna preparada en huevo embrionado (1941), que resultó fácil de preparar y con pocos efectos secundarios. ${ }^{10,11}$

En 1945, Rose et $\mathrm{al}^{12}$ demostraron la efectividad del ácido para aminobenzoico en el tratamiento, y a partir de 1948 con el desarrollo de la antibióticoterapia se eliminó la FMMR de la lista de enfermedades peligrosas.

En años recientes, se vuelve a poner en evidencia la importancia de un diagnóstico rápido y certero para esta enfermedad, no tanto por la morbilidad, sino más bien por la alta mortalidad debida a un retraso en el tratamiento o ausencia de tratamiento, al no considerar un cuadro clínico por FMMR a tiempo. 


\section{Agente infeccioso}

La Rickettsia rickettsii representa el prototipo de las bacterias que causan las fiebres manchadas. Son parásitos obligados, Gram - negativos, cocoides en forma de bastoncillo (pleomórficos), miden 0.3-0.5 x 0.8-2.0 $\mu \mathrm{m}$. y frecuentemente se observan rodeados de un halo similar al de los neumococos. Se dividen por fisión binaria en el citoplasma y el núcleo de las células infectadas de garrapatas, huevo embrionado y mamíferos. Tienen una pared celular que contiene lipopolisacáridos, péptidoglucano, unas proteínas de membrana la $O m p B$ de $190 \mathrm{kDa}$. y la $O m p A$ de $135 \mathrm{kDa}$, las cuales contienen un número variable de unidades casi idénticas, repetidas en tandem y una lipoproteína de $17 \mathrm{kDa}$. La proteína $O m p A$ es muy similar y se conserva entre las diferentes rickettsias del grupo de la fiebre manchada, sin embargo, pequeñas variaciones las caracterizan y les confieren especificidad de especie. ${ }^{13-15}$

Se encuentran en reservorios artrópodos por lo menos durante una parte de su ciclo celular, donde se mantienen por transmisión transovárica o por transmisión horizontal, en hospederos mamíferos. ${ }^{10}$

En humanos, las células blancas son las endoteliales y se diseminan vía hematógena. El foco de infección aumenta conforme pasan de célula a célula formando una red de células endoteliales infectadas en la microcirculación en dermis, cerebro, pulmones, corazón, riñones, hígado, testículos, bazo y músculo. ${ }^{18,19}$

\section{Epidemiología}

La FMMR es una enfermedad bacteriana transmitida por garrapatas de los géneros Dermacentor andersoni, $D$. variabilis y Ammblyoma americanum en los Estados Unidos $\mathrm{y}$, recientemente, se documenta infestación con una garrapata de perros, Rhipicephalus sanguineus, en sitios endémicos de FMMR. ${ }^{20,21}$ En otros países de Suramérica se han descrito garrapatas del género Amblyomma sp, tales como A. cooperi y $A$. cajennensi, en Brasil. ${ }^{22,23}$

En Costa Rica se aisló $R$. rickettsii de la garrapata de conejos Haemaphysalis leporispalustris, ${ }^{24}$ esta garrapata presenta baja preferencia por humanos y no parece tener un papel importante en la transmisión de la enfermedad, pero sí como reservorio en la naturaleza. En un estudio de campo realizado en Limón, ${ }^{25}$ se demuestra una alta densidad de formas larvales de Ammblyoma spp alrededor de las viviendas de los casos reportados, y la presencia de A.ovale en perros de la zona. La presencia de A. cajennense, de amplia distribución en Costa Rica e implicada como vector en otros países latinoamericanos, deberá evaluarse como posible vector en el país, así como la garrapata Rhipicephalus sanguineus, la garrapata del perro en la región neotropical y presente en Costa Rica. ${ }^{25}$

En 2004 se describe una nueva rickettia causal de Fiebres Manchadas en Estados Unidos, ${ }^{26}$ la Rickettsia parkeri, transmitida por $A$. maculatum. y en Uruguay se describe la transmisión de esta rickettsia por $A$. triste ${ }^{27}$ Otra rickettsia R.felis se ha reportado como agente causal de fiebre manchada transmitida por pulgas de gato, $y$ ha sido diagnosticada en México, EEUU, Brasil y países europeos, ${ }^{28}$ pero aún no existe evidencia de que se encuentre en Costa Rica.

Estas nuevas evidencias amplían el campo para el estudio de otros posibles géneros de garrapatas involucradas en la transmisión de rickettsias y de nuevas especies de estas que causan Fiebres Manchadas en el continente y que no se habían tomado en cuenta con anterioridad.

\section{Manifestaciones clínicas}

La FMMR es considerada la más severa de las fiebres manchadas, ya que puede ser letal incluso en pacientes jóvenes y previamente sanos. Los casos fatales en general se asocian a ausencia de tratamiento o tratamiento tardío, debido a que no se reconoce la entidad clínica a tiempo. La mortalidad también se vincula con la falta de signos y síntomas considerados característicos de esta infección y la ausencia de historia de contacto con garrapatas. En la era preantibióticos la tasa de mortalidad era del 20-25\%, y aún hoy, cuando existen antimicrobianos muy efectivos, la tasa es del 4- 10\%; de ahí que la recomendación mundial es considerar siempre una rickettsiosis en caso de fiebre $\mathrm{y}$ exantema maculopapular. ${ }^{29-31}$

El tiempo de incubación varía de 3-12 días, con una media de 6-8 días. Un periodo de incubación más corto suele asociarse a infecciones más severas. El cuadro clínico inicia abruptamente con cefalea intensa, mialgias generalizadas, escalofríos, malestar general y fiebre que puede alcanzar $39-40^{\circ} \mathrm{C}$ en los primeros dos días. Estos síntomas inespecíficos pueden ir acompañados de desórdenes digestivos como náusea, vómito, diarrea, anorexia y tos, los cuales con frecuencia confunden el diagnóstico con una enterocolitis, un abdomen agudo o una neumonía. El exantema (rash) aparece en el tercer día después del inicio de los síntomas, como una lesión macular de 1-5mm, y representa el foco de la infección vascular acompañada de vasodilatación. Las lesiones pueden progresar para convertirse en maculopapulares por el edema en el endotelio vascular dañado y desarrollarse en una lesión petequial purpúrica equimótica, necrótica o gangrenosa en las formas severas de la enfermedad (Figuras 1 y 2). ${ }^{29,30}$

El diagnóstico clínico durante la infección aguda es muy difícil, debido a que la triada considerada clásica de: fiebre, exantema y contacto con picadura de garrapata no siempre se presenta en los pacientes. Se ha descrito que solo el 3\% de pacientes presentan triada en los primeros 3 días de enfermedad, exantema solo en el $14 \%$ el primer día, en el 49\% en los primeros 3-5 días, en el 20\% después del sexto día y en un $9-16 \%$, nunca. Exantema en plantas y palmas, considerados típicos de FMMR ocurre entre el 36-82\% y al 


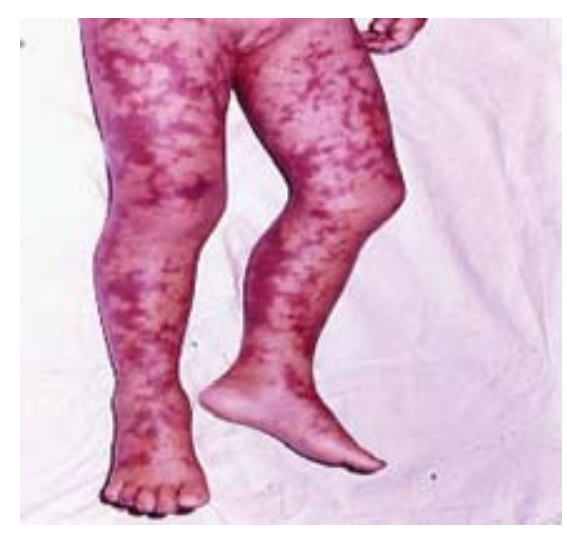

Figura.1. Caso clínico niña de Sarapiquí 1987. (Foto cortesía del Dr. Jorge Piza E. Patólogo HNN)

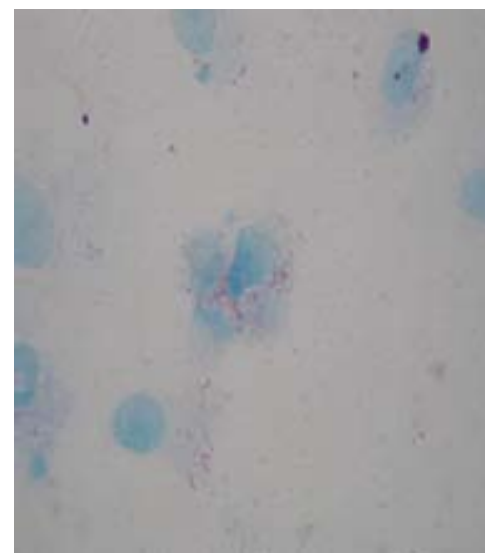

Figura.3. Patogénesis de R. rickettsii (cortesía Dra. Ximena Cortés)

quinto día solo en el $43 \%$. Petequias ocurren en $41-59 \%$ y después del sexto día en el $74 \%$ de los casos. ${ }^{29}$

Los mismos eventos patogénicos que se manifiestan en piel ocurren en todos los órganos con focos infecciosos. Los daños en el SNC, como encefalitis con confusión y letargia, ocurren en el 26-28\%; estupor y delirio en el 21-28\%; ataxia en el 18\%; coma, en el 9-10\%; convulsiones, en el $8 \%$. Las consecuencias de una FMMR pueden ser secuelas en el largo plazo, como: pérdida auditiva, vértigo, disartria, hemiplejia, paraplejia, parálisis, insuficiencia renal por hipotensión hipovolémica y baja filtración glomerular. ${ }^{1,29,30}$

\section{Patogénesis}

Las rickettsias ingresan a la célula a través de la dermis por una picadura de garrapata, y se diseminan vía sanguínea hacia el endotelio vascular. La multiplicación de las rickettsias en múltiples focos del endotelio de la microcirculación en cada órgano ocasiona un aumento en la permeabilidad vascular, con consecuentes edema e hipovolemia. El edema puede ser muy peligroso en pulmón y cerebro, ya que los fluidos intersticiales no pueden eliminarse vía linfática y la hipovolemia puede causar disfunción renal. Las lesiones multifocales en cerebro causan

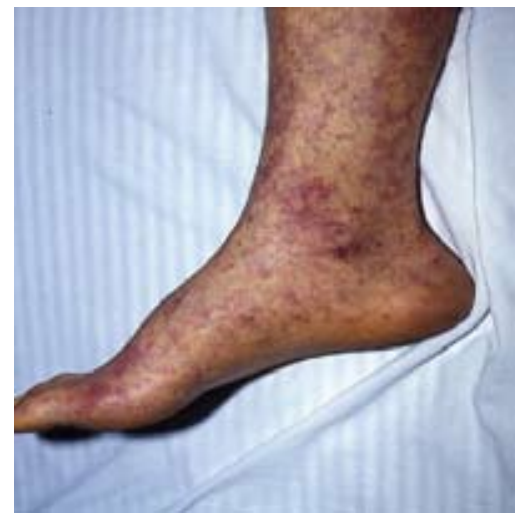

Figura.2. Caso clínico niño de Limón 1982 (Foto. Dr. L. Fuentes. Lab. Virología, Fac. Microbiología. UCR.

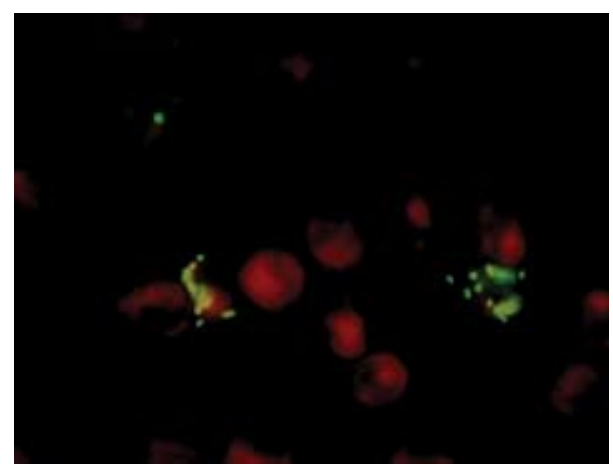

Figura.4. Tinción de Giménez. Aislamiento de R.rickettsii en células Vero. (Lab. Virología, Facultad de Microbiología. UCR).

pérdida de la función neurológica, dependiendo del área del cerebro involucrada, sin embargo, en los demás órganos las lesiones rara vez causan fallo orgánico, por ejemplo, en el hígado la destrucción de hepatocitos puede elevar las transaminasas, pero no se presenta fallo hepático. ${ }^{32,33}$

En una rickettsiosis severa, además de la hipovolemia y el edema, se postula que una oclusión trombótica y la coagulación intravascular diseminada (CID) podrían ser importantes mecanismos de patogénesis en un cuadro severo, pero en estudios en animales y en autopsias de casos fatales por $R$. rickettsii no se demuestra CID. ${ }^{32}$ Resulta interesante que los mecanismos homeostáticos de la coagulación puedan prevenir una hemorragia o la formación de trombos correctamente en una enfermedad con una infección sistémica del endotelio vascular. ${ }^{32}$ El aumento en la permeabilidad vascular es causado por la infección de las células del endotelio vascular y de los macrófagos y el daño directo con la liberación de mediadores y citoquinas.

Las rickettsias se unen al receptor celular $\mathrm{Ku} 70$ de células endoteliales a través de las proteinas OmpB y Omp A. La adherencia de la bacteria a la célula hospedera es el primer paso en la patogénesis. Inicialmente, se relacionó la adhesina $O m p A$, ya que anticuerpos contra esta proteína 
inhiben la adherencia (Figura 3). En tiempos recientes se demostró que también la Omp B está relacionada con la adherencia (Figura 3). Si bien se ha identificado la proteína $\mathrm{Ku} 70$ como uno de los receptores celulares y se conoce que las células endoteliales son el blanco principal in vivo, las rickettsias pueden infectar una gran cantidad de líneas celulares in vitro, de ahí se desprende que el receptor celular para estas bacterias es ubicuo o que las rickettsias pueden unirse a diferentes receptores. ${ }^{13,32,35}$

La adhesión de OmpB a la membrana celular induce la expresión de más Ku70, permitiendo mayor adhesión de OmpB y, consecuentemente, mayor infección. Esta unión también induce mayor reclutamiento de ubiquitina ligasa, lo que posibilita la internalización del complejo OmpA(B)/Ku70 e inhibe la degradación proteosomal, y como consecuencia la célula no entra en apoptosis. ${ }^{34,35}$ Esta modificación postransduccional conlleva al reclutamiento del complejo Arp2/3, el cual es activado por tirosín quinasas de la familia src, lo que permite la fagocitosis de las rickettsias por un mecanismo de zipper que involucra una alteración del citoesqueleto de actina en el sitio de entrada. ${ }^{34-36}$

Otra proteína codificada por las rickettsias es la Rick A, la cual se expresa en la superficie de la bacteria y es la responsable de la activación del complejo Arp2/3 para la polimerización de las actinas celulares. Estos filamentos de actina propalan (filipodia) a las rickettsias hacia la superficie celular, la membrana citoplasmática se invagina dentro de la célula adyacente y la fusión de ambas membranas celulares permite el paso de las rickettsias de célula a célula, sin pasar por el espacio extracelular (Figura 3). ${ }^{37}$

Otras rickettsias como la R.typhi no estimulan la movilidad por filamentos de actina, sino que se acumulan en grandes cantidades dentro de la célula hasta que esta estalla y libera las bacterias al torrente sanguíneo. ${ }^{33}$

Las rickettias escapan de la membrana del fagososoma porque liberan fosfolipasa $\mathrm{D}$ y hemolisina $\mathrm{C}^{33}$, las cuales la destruyen y son liberadas al citosol donde se multiplican por fisión binaria (Figura.3). La invasión de $R$. rickettsii del citoplasma al núcleo produce el daño y muerte de estas células, y como resultado se produce la extravasación de fluidos del endotelio a otros órganos, causando encefalitis, edema pulmonar, arritmias cardiacas y sangrado. ${ }^{33}$

Recientemente, Walker ${ }^{38}$ plantea la posibilidad de que la actividad de polimerización de la actina pueda interrumpir un elemento de la barrera endotelial, que son las uniones adherentes de cuya estabilidad depende la integridad del citoesqueleto de actina. Se observó que la infección de células endoteliales con $R$. conorii está asociada con el desarrollo de discontinuidades en la localización de las proteínas de las uniones adherentes (gaps o brechas intercelulares) durante el curso de una infección in vitro. Estas observaciones aunadas a un cambio en la morfología celular de poligonal a fusiforme, ${ }^{32,38}$ la inconsistencia de la localización de las rickettsias adyacentes a las brechas interendoteliales y la simultánea formación de fibras de actina, sugieren que los cambios en la localización y las características de tinción de las proteínas de las uniones adherentes no son el resultado directo de la destrucción del citoesqueleto de actina por las rickettsias. Se plantea las hipótesis de que el desarrollo de las brechas interendoteliales durante la infección es el resultado de dos fenómenos: un desnudamiento del endotelio seguido de un proceso fisiológico de respuesta de reparación y una respuesta inflamatoria como consecuencia de la activación de los receptores a través de señales paracrinas, o como resultado de la activación de la trascripción de NF-kB mediada por la bacteria. Esta proteína puede dirigir la activación de varias vías de inflamación. ${ }^{38,39}$ Interesantemente, este efecto ocurre tanto como consecuencia de la unión de los mediadores de la inflamación a los receptores endoteliales como por la adhesión de leucocitos al endotelio. ${ }^{39}$

Se ha discutido sobre diferencias en el grado de virulencia entre las cepas de $R$. rickettsii comparando su patogenicidad en conejillos de indias y en humanos, sin embargo, no queda claro si existen realmente diferencias estructurales importantes que puedan explicar tales variaciones. En estudios comparativos de las secuencias Omp A, Omp B de cepas de R.rickettsii aisladas en distintas regiones del mundo y de hospederos variados, que presentan mayor o menor virulencia durante la infección, no se ha podido encontrar características que puedan explicar esta diferencia de comportamiento. ${ }^{40-42}$

\section{Respuesta inmune}

Las células NK se activan muy al inicio de la infección e inhiben el crecimiento de las rickettsias en asociación con la producción de IFN- $\gamma$. La eliminación de las rickettsias del organismo la realizan los linfocitos T citotóxicos (CD8+), que reconocen específicamente las células infectadas. Los anticuerpos contra Omp A y B aparecen cuando la infección está controlada y hay recuperación; estos son protectores contra reinfecciones. Tanto los linfocitos CD4+ como los CD8+ contribuyen con la producción de citoquinas que activan las células endoteliales. Las células endoteliales activadas por IFN- $\gamma$, TNF- $\alpha$, IL-1 $\beta$ y RANTES eliminan las rickettsias intracelulares por dos mecanismos bactericidas: la producción de oxido nítrico y peróxido de hidrógeno. .2-34,43-44 $^{-1}$

La Rickettsia rickettsii es una bacteria que causa una enfermedad muy severa en humanos, cuyo tropismo y multiplicación en las células del endotelio vascular se asocia con efectos citopáticos que contribuyen con el aumento en la permeabilidad vascular. Las células endoteliales infectadas responden a la infección alterando la expresión de varias proteínas, tales como: factor tisular, factor inhibidor de plasminógeno, selectina E, interleucina-1 (IL-1), interleucina -6 (IL-6) e interleucina -8 (IL-8). Los estudios ultraestructurales demuestran un daño severo en las células evidenciado por la dilatación del retículo endoplásmico 
rugoso, pérdida del control de la osmolaridad y lisis celular. $^{45}$

Con base en los cambios en el citoesqueleto de las células infectadas se propone que $R$. rickettsii es una bacteria oxígenorreactiva, la cual induce acumulación intracelular de radicales de peróxidos y superóxidos, de peróxido de hidrógeno extracelular, y causa la disminución en los niveles intracelulares de tioles. ${ }^{46,47}$ La peroxidación de los lípidos de la membrana inducida por radicales de oxígeno o radicales orgánicos, se genera por el metabolismo de los compuestos presentes en el medio de cultivo celular. Estos radicales, producidos normalmente por la célula, poseen un mecanismo de regulación que controla su producción para evitar daño celular. Las actividades del sistema antioxidante están reguladas por tres enzimas: la glucosa-6 fosfato deshidrogenasa, la catalasa y la glutatión peroxidasa, las cuales disminuyen durante una infección por R.rickettsii, ${ }^{17}$ mientras que la enzima superóxido dismutasa aumenta. Se ha demostrado que el ácido alfa -lipoico es un potente antioxidante y tiene efecto protector contra cambios oxidativos, al disminuir los niveles de peróxidos y elevar los niveles de glutatión reducido y la actividad de la glutatión peroxidasa en células infectadas por $R$. rickettsii. Estudios recientes demuestran que las células endoteliales infectadas inducen la expresión de la enzima heme-oxigenasa -1(HO1), como mecanismo protector contra el daño oxidativo, y es vasoprotector; ${ }^{45}$ en otra investigación se evidencia que el mecanismo regulador de la función HO-1 en la vasculatura es el control de la actividad del sistema de la ciclooxigenasa (COX), ${ }^{16}$ responsable de generar substancias vasoactivas, como las prostaglandinas, prostaciclinas y tromboxano. Estos resultados demuestran el estrés oxidativo que se produce en las células infectadas por $R$. rickettsii y explican el aumento en la permeabilidad vascular como parte de los daños que causa el cuadro clínico tan severo.

\section{Diagnóstico de laboratorio}

Los hallazgos de laboratorio más frecuentes son: trombocitopenia, trastornos hidroeletrolíticos (hiponatremia, hipokalemia) y elevación de aspartato aminotransferasa (AST) y alaninatransferasa (ALT).

La mayoría de los laboratorios en el mundo no realizan pruebas diagnósticas para R.rickettsii ni otras rickettsiosis. Por muchos años se utilizó la prueba de Weil Felix, por la reacción cruzada que presenta la Rickettsia rickettsii con antígenos de Proteus OX-19. Los anticuerpos aglutinantes en la prueba de Weil Felix se detectan a los 5-10 días después del inicio de los síntomas y casi conjuntamente con la aparición de la IgM. Sin embargo, se han descrito casos de una reacción de Weil Felix positivo en ausencia de IgM, por lo que se ha demostrado una muy baja especificidad y sensibilidad de esta prueba para el diagnóstico de FMMR. ${ }^{29,48}$
Actualmente, para el diagnóstico de laboratorio de una FMMR se emplean tres estrategias: serología, cultivo e identificación, inmunohistoquímica y PCR.

Los ensayos serológicos son los más utilizados para confirmar los casos de FMMR por inmunofluorescencia indirecta (IFA), y el Centro para enfermedades infecciosas los considera la prueba estándar de referencia. Se puede detectar IgM o IgG. Para un diagnóstico específico se debe contar con sueros pareados: el primero obtenido durante la infección aguda y el segundo de dos a tres semanas, después para demostrar la seroconversión. La IgG generalmente aparece de 7-10 días después del inicio del cuadro clínico. En caso de contar con anti IgM humana marcada, el diagnóstico sería más rápido con un $94 \%$ de especificidad y sensibilidad. ${ }^{49}$

El aislamiento y la identificación de rickettsias son procedimientos difíciles y laboriosos. Muy pocos laboratorios los efectúan, ya que requieren de equipos de seguridad tipo 2-3 y personal con experiencia. Durante muchos años el aislamiento se realizó inoculando intraperitonealmente conejillos de indias machos de $400 \mathrm{~g}$ con muestras de sangre total, coágulos plasma, muestras de biopsias y de garrapatas. Una vez identificada la infección en el cuilo, evidenciada por fiebre alta, pérdida de peso y necrosis testicular, se deben hacer pasajes en otros cuilos o inocular huevos embrionados en saco vitelino con el fin de obtener suficientes bacterias para su posterior identificación. La presencia de las rickettsias se determina inicialmente haciendo frotis por aposición de los sacos vitelinos infectados y con tinción de Giménez ${ }^{50}$ (Fig.4). La identificación final se realiza por medio de inmunofluorescencia con anticuerpos específicos contra $R$. rickettsii (Figura 5). ${ }^{51}$

En los últimos años se han desarrollado sistemas de cultivos celulares para el aislamiento e identificación de rickettsias y actualmente se utilizan para su cultivo muchas

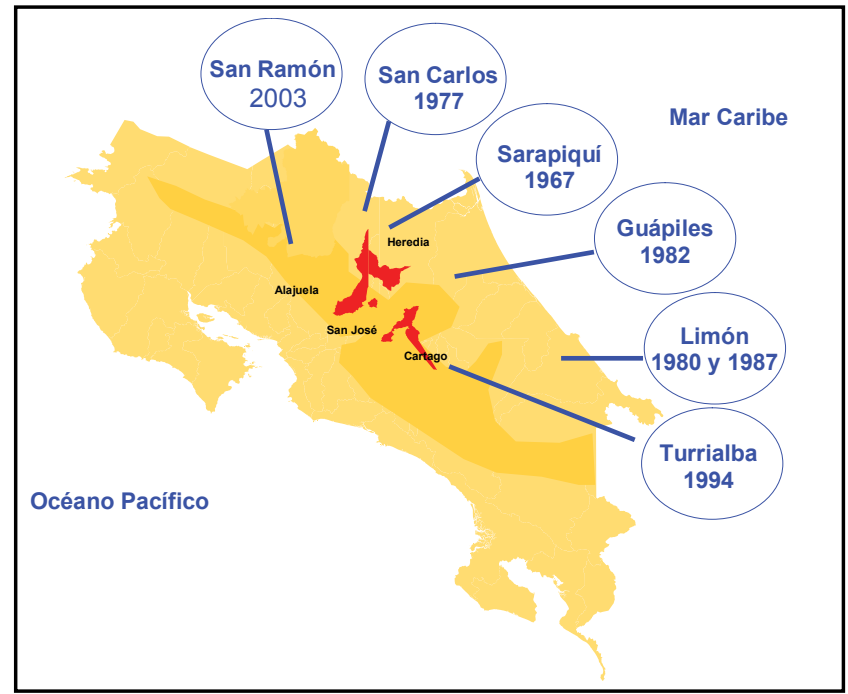

Figura.5. Identificación de aislamiento de R.rickettsii en células Vero por Inmunofluorescencia directa. (Lab. Virología. Facultad de Microbiología. UCR). 
líneas celulares. Las células Vero y las L929 de ratón son las más sensibles para aislar rickettsias de muestras con muchas bacterias. Se deben hacer frotis y, tinción de Giménez cada dos días en procura de para determinar el crecimiento de las bacterias y, generalmente, a los 8-10 días se tiene un crecimiento confluente. Las células HEL (diploides humanas de pulmón embrionario) se usan para la técnica de Shell vial, lo que permite un diagnóstico más rápido de la infección, en alrededor de 24-48 horas. La presencia de las rickettsias en ambos casos se determina con tinción de Giménez y la identificación específica por IFA. ${ }^{52}$

La inmunohistoquímica es utilizada especialmente en biopsias de piel de pacientes con brote cutáneo antes del inicio del la antibioticoterapia, y por lo menos antes de 48 horas con terapia. Dado que las rickettsias se distribuyen focalmente en los tejidos en una FMMR, no siempre se detecta el agente, aún en los laboratorios más experimentados; la sensibilidad del método es de un $70 \%$. Este método también se puede utilizar para cortes de tejido de bazo, hígado y otros obtenidos en autopsias de pacientes que fallecieron con sospecha de FMMR. ${ }^{53}$

Recientemente se ha desarrollado un método molecular más rápido, seguro, específico y sensible, que consiste en detectar el ADN bacteriano y amplificar secuencias seleccionadas para la reacción en cadena de la polimerasa (PCR). Regnery ${ }^{33,54}$ propuso en 1991 la amplificación del gene que codifica para $\operatorname{Omp} A$, lo que permite la diferenciación entre 9 rickettsias del grupo de las fiebres manchadas. En 1994 Ermeeva $^{55}$ utiliza una combinación de secuencias de $O m p A$ у $O m p B$ y, posteriormente, Cabruna ${ }^{22}$ introduce el gen para la citrato sintetasa glt $A$. Con esta metodología se hace un dianóstico específico de la R.rickettsii y además se permite caracterizar las cepas por secuenciación. En ninguno de los estudios realizados hasta la fecha se ha podido determinar diferencias significativas en las secuencias de ADN de R.rickettsii aisladas en diferentes regiones del mundo de humanos, perros y garrapatas. ${ }^{56}$

\section{Tratamiento}

El tratamiento con los antibióticos apropiados debe iniciarse inmediatamente cuando hay sospecha de una FMMR con base en los hallazgos clínicos y epidemiológicos. No conviene esperar el diagnóstico de laboratorio para iniciarlo. El paciente generalmente responde 24-72 horas después de iniciado el tratamiento; si no lo hace, debería sospecharse de otro agente etiológico. La tetraciclina (Doxycycline) es la droga más recomendada por la evidencia clínica y experimental de su mayor efectividad frente al cloramfenicol. Solo en mujeres embarazadas se recomienda el uso del cloramfenicol para evitar malformaciones asociadas con tetraciclinas en los dientes y huesos de los bebés. El cloramfenicol se asocia con otros efectos secundarios, como la anemia aplástica, por lo que se recomienda monitoreo sanguíneo constante. ${ }^{1,31,57}$

\section{Prevención y control}

Evitar contacto con garrapatas es la manera más efectiva de evitar una infección transmitida por estos vectores. Las personas expuestas a estos artrópodos deben inspeccionarse rápidamente y eliminar las posibles garrapatas unidas al cuerpo ya que la infección puede tardar horas desde que la garrapata se pega hasta que transmita los microorganismos. No existe en estos momentos una vacuna aprobada para prevenir la FMMR.

Dado que es muy difícil pensar que las personas pueden evitar el contacto con garrapatas en paseos, zonas rurales y en ocupaciones de riesgo (veterinarios, técnicos, etc), se sugiere que: utilicen ropa clara para detectar la presencia de las garrapatas, no dejen descubiertas partes de la piel entre los pantalones y las medias y usen repelentes que contengan permitrina en la ropa y zapatos, ya que permanece por muchos días.

Los repelentes que contienen DEET (dietiloalbutamina) pueden aplicarse en la piel, su efecto dura unas horas, pero dado que es necesario varias aplicaciones durante el día. El uso en niños, en grandes cantidades, se asocia con efectos secundarios.

Se debe realizar una revisión completa del cuerpo una vez que se regresa del paseo, con el propósito de buscar de garrapatas pegadas al él, es importante utilizar lupas para observar las garrapatas pequeñitas (mostacilla). Como recomendación, es fundamental guardar las garrapatas encontradas en un frasco limpio, ya que sería de mucha utilidad contar con esa información y muestra para un diagnóstico más preciso, en caso de que se presente un cuadro clínico asociado. ${ }^{5,29}$

\section{Rickettsiosis en Costa Rica}

1952- Primer informe de rickettsiosis humanas en Costa Rica. ${ }^{3}$; 1971-Estudios realizados con sueros deCentroamérica reportan serología positiva en los sueros procedentes de Costa Rica y Honduras. ${ }^{3}$

1975- Cedral de San Carlos: Primer brote de FMMR reportado en Costa Rica ${ }^{3}$ con dos casos, uno de ellos fatal. En el Laboratorio de Virología de la Facultad de Microbiología se realiza el aislamiento del agente etiológico a partir de coágulos de sangre de los pacientes e inoculados en cobayos. Posteriormente, se efectúan pasajes en huevo embrionado y se detecta la presencia del agente por tinción de Giménez. Los estudios de los sueros de los pacientes demostraron seroconversión para el grupo de las fiebres manchadas por fijación de complemento y fueron confirmadas después por inmunofluorescencia.

La identificación del agente etiológico se realizó por inmunofluorescencia en el Laboratorio de la Montañas Rocosas, Montana, Estados Unidos y demostró ser Rickettsia rickettsii, indistinguible de las cepa prototipo Sheila Smith. 
1977- Se iniciaron estudios epidemiológicos y clínicos, se describió la enfermedad en el país y se desarrollaron los métodos para el aislamiento de estas bacterias en cobayos, huevo embrionado y cultivos celulares, así como la detección de rickettsias por tinción de Giménez e identificación por inmunofluorescencia ${ }^{24,25,59}$

1980-Puerto Limón: Se presenta 1 caso fatal, se aísla el agente y se identifica como $R$. rickettsii. Los estudios serológicos de la familia demostraron dos casos asintomáticos con seroconversión para esta bacteria ${ }^{24}$.

1985-Zona atlántica: Se aísla e identifica R. rickettsii de las garrapatas Haemaphysalis leporispalustris del conejo silvestre Sylvilagus braziliensis ${ }^{24}$.

1986- Franja al norte de la Cordillera Volcánica Central: Estudios ecológicos y seroepidemiológicos realizados demuestran seropositividad contra $R$. rickettsii en sueros de conejos silvestres en las zonas de Cedral de San Carlos, Jiménez y Puerto Limón. Se demuestra seropositividad en sueros de personas en Cedral, en Jiménez y en Limón y se reporta seropositividad en perros de Cedral, Jiménez, Matina y Limón ${ }^{59}$. Es importante recalcar que hasta la fecha no se han reportado casos humanos en Jiménez

1987- Puerto Limón y Sarapiquí: Dos casos fatales en Limón y un caso fatal en Sarapiquí. Se aísla e identifica el agente etiológico en todos los casos y se confirma por seroconversión $^{25}$.

1994-Turrialba: Cinco casos fatales, por ser una zona no reconocida anteriormente como endémica para FMMR; en un inicio las autoridades de salud sospecharon de otros agentes etiológicos y luego se demostró por serología en el laboratorio que se trataba de una rickettsiosis, lo cual fue confirmado posteriormente en el CDC por PCR.

2003-San Ramón: 5 casos, tres hospitalizaciones, ninguno fatal. Las muestras fueron enviadas prontamente por el Dr. Roberto Marín, jefe del Laboratorio del Hospital de San Ramón; se aisló e identificó $R$. rickettsii (Figura 6) y se demostró la seroconversión en los 5 casos, lo cual fue posteriormente confirmado por CDC.

Recientemente se hizo un estudio de caracterización genómica de todas las rickettsias aisladas en Costa Rica y se evidenció que no hay diferencias significativas entre las cepas costarricenses de humanos y de garrapatas comparadas con la cepa prototipo Sheila Smith. ${ }^{60}$ Es interesante recalcar que la cepa de $R$. rickettsii aislada de garrapatas de conejo Haemophysalis leporispalustris en Costa Rica se comportó muy virulenta cuando se infectaron cobayos, ${ }^{24}$ lo que contradice otros estudios de bacterias aisladas de esta misma

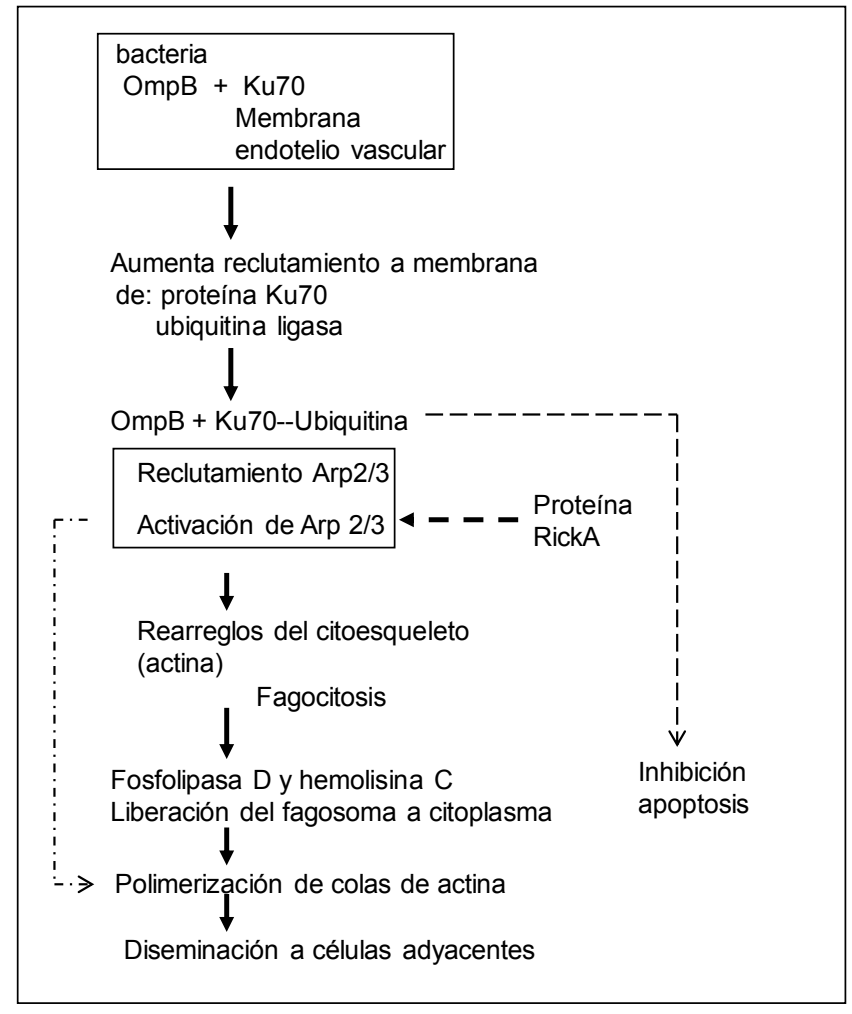

Figura.6. Brotes de R.rickettsii en Costa Rica.

especie de garrapata. Sin embargo, no fue posible demostrar diferencias en las secuencias de ADN estudiadas con las de otras garrapatas.

Otro aspecto importante de recalcar es el estudio seroepidemiológico de toda la familia relacionada con el caso fatal en Limón en 1982, en el cual se demuestra seroconversión semejante a la del paciente, tanto en los padres del joven como en un hermano, todos ellos asintomáticos; esto contradice algunos datos de la bibliografía donde se afirma que no hay casos asintomáticos de la infección y coincide con estudios recientes en donde se demuestran resultados semejantes en un grupo familiar. ${ }^{58,59,61}$

\section{Agradecimientos:}

Un merecido reconocimiento al Dr. Luis Guillermo Fuente Leiva, Profesor Benemérito "in memoriam" de la Facultad de Microbiología, pionero en el estudio de las rickettsiosis en Costa Rica.

Un especial agradecimiento a las siguientes personas, por su colaboración en la lectura del documento y sus valiosos aportes: Dra. Lizeth Taylor, Dr. Cesar Rodríguez y Dra. Ximena Cortés, quien además diseñó el cuadro de patogénesis. 


\section{Referencias}

1. Masters EJ., Olson GS, Scott. JW, Paddock CD. Rocky Mountain Spotted Fever. A clinician's dilemma. Arch. Intern. Med.2003; $16 ; 3769-774$.

2. Dumier JS, Walker DH. Rocky Mountain Spotted Fever- Changing Ecology and Persisting Virulence. N. Engl. J. Med.2005;353; 551553.

3. Fuentes LG. Primer caso de Fiebre de las Montañas Rocosas en Costa Rica, América Central. Re.Lat-amer.Microbiol.1979;21;167-172.

4. Fournier PE, Raoult D. Identification of rickettsial isolates at the species level using multi-spacer typing. BMC Microbiology 2007;7:127.

5. Ricketts HT. Some aspects of Rocky Mountain Spotted Fever as shown by recent investigations. Rev. Infect. Dis.1991;13:1227-40. (Reprinted from the Medical Record 1909;76:843-55)

6. Wilson LB, Chowning WM. Studies in Pyroplasmosis Hominis ("Spotted Fever or Tick Fever" of the Rocky Mountains) . J. Infect. Dis. 1904; 1:31-57,

7. Badger LF., Dyer RE., Rumreich A. An infection of the Rocky Mountain Spotted Fever. Identification in the eastern part of the United States. Public Health Reports. 1931;1452:463-470.

8. Weiss E, Strauss BS. The life and career of Howard Taylor Ricketts. Rev.Infect.Dis. 1991;13:1241-1242.

9. Horsfall FL,Tamm I. editors. Viral and rickettsial Infections of man. 1965. J.B. Lipincott Co. Fourth edition.

10. McDade JE., Newhouse VF. Natural History of Rickettsia rickettsii. Ann.Rev.Microbiol.1986; 40:287-309.

11. Philip RN. Historical ruminations: Rickettsiae and the Rocky Mountain Laboratory. Ann. N. Y. Acad. Sci. 1990;590:1-9.

12. Rose H.M., Duane RB., Fischel EE. The treatment of spotted fever with para -aminobenzoic acid.JAMA. 1945;129:1160-1161.

13. Gilmore Jr RD., Clepak Jr.W., Policastro PF.,Hackstadt T. The 120 kilodalton outer membrane protein $(\mathrm{rOmpB})$ of Rickettsia rickettsii is encoded by an unusually long open reading frame: evidence for protein processing from a large precursor. Molecular Microbiology.1991;5:2361-2370.

14. Gilmore RD. Comparison of the rOmpA gene repeat regions of Rickettsia reveals species specific arrangement of individual repeating units.Gene.1993; 125: 97-102.

15. Policastro PF, Hackstadt T. Differential activity of Rickettsia rickettsii ompA and omp B promoter regions in a heterologous reporter gene system. Microbiology.1994;40:2941-2949.

16. Xu W.,Raoult D. Distribution of immunogenic epítopes on the two major immunodominant proteins (rOmpA and rOmpB) of Rickettsia conorii among the other Rickettsiae of the Spotted Fever Group. Clinical and Diagnostic Laboratory Immunology. 1997; 4:753-763.

17. Fournier PE., Roux V., Raoult D. Phylogenetic analysis of spotted fever group rickettsiae by study of the outer surface protei rOmpA. International Journal of Systematic Bacteriology.1998; 48:839-849.

18. Rydkina E.,Sahni A.,Baggs RB.,Silverman DJ.,Sahni SK. Infection of human endothelial cells with spotted fever group Rickettsiae stimulates Cyclooxygenasa 2 expression and release of vasoactive prostaglandins. Infection and Immunity.2006;74:5067-5074.

19. Palaniyandi S., Devemanohran L, Santucci LA., June EH., Xiaojingang T., Silverman DJ. Infection of Human endothelial cells by Rickettsia rickettsii causes a significant reduction in the levels of key enzymes involved in protection against oxidative injury. Infection and Immunity.1994; 62:2619-2621.

20. Kidd L., Hegarty B., Sexton D., Breitschwerdt E. Molecular Characterization of Rickettsia rickettsii infecting dogs and people in North Carolina. Ann.N.Y.Acad.Sci. 2006;1078: 400-409.
21. Nicholson WL., PaddockCD., Demma L., Traeger M., Jonson B., Dickson J., McQuinston J., Swerdlow D. Rocky Mountain Spotted Fever in Arizona: Documentation of heavy environmental infestation or Rhipicephalus sanguineus at an endemic site. Ann.N.Y.Sci. 2006; 1078:338:341.

22. Labruna MB., Whitworth T., Horta MC., Bouyer DH., McBride JW., Pinter A., Popov V., Gennari SM., Walker D. Rickettsia species infecting Amblyomma cooperi ticks from an area in the State of Sao Paulo, Brazil, where Brazilian Spotted Fever is endemic. J. Clin. Microbiol. 2004; 42:90-98.

23. Sangioni,LA., Horta MC., Vianna MCB., Gennari SM., Soares RM., Galvao MA., Schumaker T., Ferreira F.,Vidotto O. Labruna MB. Rickettsial infection in animals and Brazilian Spotted Fever endemicity. Emerging Infectious Diseases.2005;11:265-270.

24. Fuentes L., Calderón A, Hun L.. Isolation and identification of Rickettsia rickettsii from the rabbit tick Haemophysalis leporispalustris in the Atlantic zone of Costa Rica. . Am. J. Trop. Med. Hyg 1985; 34: 564:567.

25. Hun L., Herrero L., Fuentes, L.,Vargas, M. Tres nuevos casos de Fiebres Manchadas de las Montañas Rocosas en Costa Rica. Rev. Cost. Cienc. Med. 1991;12:51-56.

26. Paddock CD., Summer JW., Comer JA., Zaki SR., Goldsmith CS., Goddard J., McLellan S., Tamminga C., Ohi CA. Rickettsia parkeri: A newly recognized cause of Spotted Fever Rickettsiosis in the United States. CID. 2004;38:805-811.

27. Venzai JM., PortilloA., Estrada Peña A., Castro O.,Cabrera PA., Oteo JA. Rickettsia parkeri in Amblyoma triste from Uruguay. Emerging Infectious Diseases. 2004;10:1493-1495.

28. Horta MC., Labruna MB., Durigon.EL., Schumaker TS. Isolation of Rickettsia felis in the mosquito cell line C6/36. Appl Environ Microbiol.2006;72:1705-1707.

29. Walker D. Rocky Mountain Fever: A season alert. CID.1995;20:11111117.

30. Lacz NL., Schwartz RA., Kapila r. Rocky Mountain Spotted Fever. JEADV.2006;20:411-417.

31. Holman RC., Paddock CD., Curns AT.,Krebs JW, McQuiston JH.,Childs JE.J. Analysis of risk factors for fatal Rocky mountain Spotted fever: Evidence for superiority of tetracyclines for therapy. J. Infect.Dis.2001;184:1437-1444.

32. Li H.,Walker DH. rOmpA is critical for the adhesion of Rickettsia rickettsii to host cells. Microb Pathog. 1998;24:289-298.

33. Walker DH., Valbuena GA., Olano JP. Pathogenic mechanisms of diseases caused by Rickettsia. Ann.N.Y.Acad.Sci. 2003;990:1-11.

34. Walker D. Rickettsiae and Rickettsial Infections: The current state of knowledge. Clin.Infec.Dis.2007;45(Suppl 1):39-44.

35. Martinez JJ., Seveau S., Veiga E., Matsuyama S., Cossart P. Ku70, a component of DNA dependent protein kinase, is a mammalian receptor for Rickettsia conorii. Cell.2005; 123: 1013-1023.

36. Walker DH. Targeting Rickettsia. N. Engl. J. Med..2006;354:14181420.

37. Gouin E., Eglie C., Dehoux P., Villers V., Adams J., Gertier F., Li R., Cossart P. The Rick A protein of Rickettsia conorii activates the Arp2/3 complex. Nature. 2004; 427:457-461.

38. Valbuena G., Walker DH. Changes in the adherens junctions of human endothelial cells infected with spotted fever group rickettsiae. Virchows Arch. 2005; 446:379:382.

39. Sporn LA.,Sahni SK., Lerner NB.,Marder VJ., Silverman DJ., Turpin LC., Shwab AL.. Rickettsia rickettsii infection of cultures human endothelial cells induces NF- $\kappa \mathrm{B}$ activation. Infection and Immunity. 1997; 65:278627-91.

40. Anacker RL, Philip RN., Williamd JC., List RH., Mann RE. I Biochemical and Immunochemical analysis of Rickettsia rickettsii strains of various degrees of virulence. Infection and Immunity.1984; 44:559-564. 
41. McDade JE. Evidence supporting the hypothesis that rickettsial virulence factors d etermine the severity of Spotted Fever and Typhus group infections. Ann.N.Y.Acad.Sci. 1990;590:20-6.20-26.

42. Ermeeva ME., Dasch GA., Silverman DJ. Quantitative analyses of variations in the injury of endothelial cells elicited by 11 isolates of Rickettsia rickettsii. Clinical and Diagnostic Laboratory Immunology. 2001;8:788-796.

43. Walter D, Olano JP, FENA HM. Critical role of citotoxic T linphocytes in immune clearance of rickettsial infection. Infection and Immunity. 2001;69:1841-1846

44. Zavala J., Ruiz A., Zavala J. Las Rickettsias del grupo de las fiebres manchadas: Respuesta inmune y sus proteínas inmunodominantes. Rev Méd Chile. 2004; 132:381-387.

45. Rydkina E, Sahni A, Silverman D, Sahni S. Rickettsia rickettsii infection of cultured human endothelial cells induces heme-oxygenase 1 expression. Infection and Immunity. 2002; 70: 4045-4052.

46. Silverman D, Santuccii LA. Potential for free radical-induced lipid peroxidation as a cause of endothelial cell injury in Rocky Mountain Spotted Fever. Infection and Immunity. 1988;56:3110-3115.

47. Hong JE, Santucci LA, Tian X, Silverman D. Superoxide dismutasedependent, catalase -sensitive peroxides in human endothelial cells infected by R.rickettsii. Infection and Immunity.1998;66:1293-1298.

48. Paddock CD, Creer PW, Ferebee TL, Singleton Jr. J, McKechnie DB, Treadwell TA. Hidden mortality attributable to Rocky Mountain Spotted Fever: Immunohistochemical detection of fatal serologic unconfirmed disease. J.Infect.Dis. 1999;179: 1469-1478.

49. Philip RN., Casper RA., Peacock MG., Burgdorfer W. Microimmunofluorescence test for the serological study of Rocky Mountain Spotted Fever. J. Clin. Microbio. 1976;3: 51-61.

50. Giménez DF. Staining rickettsiae in yolk sac culture. Stain Technol. 1964;39:135-140.

51. La Scola B., Raoul D. Laboratory diagnosis of rickettsiosis: Current aproach to diagnosis of old and new rickettsial diseases. J. Clin. Microbiol. 1997;35:2715-2727.
52. Vestris G., Rolain PE., Fournier ML., Enea BM., Patrice JY., Raoult D. Seven years' Experience of isolation of Rickettsia spp. from clinical specimens using the shell vial cell culture assay. Ann.N.Y.Sci. 2003; 990:371-374.

53. Walker D. Rickettsia rickettsii: As virulent as ever. Am.J.Trop.Med. Hyg. 2002; 66:448-449.

54. Roux V., Fournier PE.,Raoult D. Differentiation of Spotted Fever Group Rickettsiae by sequencing and analysis restriction fragment lenghth polymorphism of PCR-amplified DNA of the gene encoding the protein rOmpA. J. Clin. Microbiol.1996;34:2058-2065.

55. Eremeeva ME., Dasch GA., Silverman DJ. Evaluation of a PCR assay for quantitation of Rickettsia rickettsii and closely related Spotted Fever Rickettsiae. J. Clin. Microbiol 2003; 41:5466-5472.

56. Karpathy SE., Dasch GA., Eremeeva ME. Molecular typing of isolates of Rickettsia rickettsii using DNA sequencing variable intergenic regions, J.Clin.Microbiol. 2007;45:2545-53.

57. Dalton JM., Clarke MJ.,Holman RC.,Krebs JW., Fishbein DB., Olson JG.,Childs JE. National Surveillance for Rocky Mountain Spotted Fever 1981-1992: Epidemiology summary and evaluation of risk factors for fatal outcome. Am.Trop.Med.Hyg.1995;52:405-413.

58. Jones TF., Craig AS., Paddock CD., McKechnie DB., Childs JE., Zaki SR., Schaffner W. Family cluster of Rocky Mountain Spotted Fever. CID. 1999; 28:853-859.

59. Fuentes LG. Ecological study of Rocky Mountain Spotted Fever in Costa Rica. Am J Trop Med Hyg. 1986; 35: 192-196.

60. Hun L, Cortes X, Taylor L. Molecular Characterization of Rickettsia rickettsii isolated from human clinical samples and from the rabbit tick Haemaphysalis leporispalustris collected at different geographic zones in Costa Rica. (enviado a publicación)

61. Fatal cases of Rocky Mountain Spotted Fever in family clusters-Three states. JAMA. 2007;292:31-33. (Reprinted from MMWR. 2004;53: 407-410). 